\title{
The spin of the nucleon from the HERMES point of view \\ D. Hasch ${ }^{\mathrm{a} *}$ (on behalf of the HERMES Collaboration) \\ ${ }^{a}$ INFN-Frascati, \\ via E. Fermi 40, 00044 Frascati (Rm), Italy
}

In its 12 years of successful running, the HERMES experiment has addressed all aspects in studying the spin structure of the nucleon by measuring not only inclusive but also semi-inclusive and exclusive processes in deepinelastic lepton scattering. Novel and pioneering results on the spin structure of the nucleon are presented which illustrate the new trends in the field. We concentrate here on the quark spin flavour decomposition of the nucleon spin, the extraction of the gluon polarisation and on novel transverse spin phenomena. The new formalism of Generalised Parton Distributions and their investigation in hard exclusive processes is discussed elsewhere at this workshop [1].

\section{Introduction}

A major goal in the study of QCD in recent years has been the detailed investigation of the spin structure of the nucleon. Vast experimental and theoretical activities were initiated by the observation of the EMC experiment that only a small fraction of the nucleon's spin can be attributed to the spins of the quarks, which became known as the 'spin crisis'. The model independent helicity sum rule

$S_{z}=\frac{1}{2}=\frac{1}{2}(\Delta u+\Delta d+\Delta s)+\Delta G+L_{z}$

decomposes the longitudinal projection of the spin into contributions from up, down and strange quark spins (summarised as $\Delta \Sigma$ ), from gluon spins $\Delta G$ and from orbital angular momentum $L_{z}$ of quarks and gluons. Here, $\Delta f=f^{+}-f^{-}$ist the difference of partons with their spin aligned $f^{+}$or anti-aligned $f^{-}$to the spin of the nucleon. HERMES aims to obtain information on all these contributions by measuring inclusive and semi-inclusive deep-inelastic scattering processes as well as hard exclusive processes.

In the following sections, after a brief description of the experiment, are presented the world data on the polarised structure function $g_{1}(x)$ of the proton and the deuteron from measurements with longitudinally polarised beams and targets

*email: delia.hasch@lnf.infn.it and the so far most precise determination of $\Delta \Sigma$. By also observing a produced hadron in coincidence with the scattered lepton it has been possible to extract the helicity distributions of individual quark flavours in the nucleon. New recent results emphasize the role of strange quarks. The polarisation of gluons is explored by measuring the photon-gluon fusion process. The pioneering results on transverse spin phenomena are closely connected to novel trends in the field that aim for a complete description of the hadron structure beyond the collinear approximation. These discussions are completed by the presentation elsewhere at this workshop [1] of HERMES results on hard exclusive reactions that relate to the new concept of Generalised Parton Distributions.

The HERMES experiment has been taking data at the HERA accelerator in Hamburg, from 1995 to 2007 . HERMES scattered longitudinally polarised electron and positron beams of 27.5 $\mathrm{GeV}$ from longitudinally or transversely polarised targets internal to the beam pipe. Featuring polarised beams and targets, and an open-geometry spectrometer with excellent particle identification, HERMES is well suited to study the spindependent inclusive and semi-inclusive DIS reactions. The PID capabilities of the experiment were significantly enhanced in 1998 when the threshold Cerenkov detector (used to identify pions above momenta of $4 \mathrm{GeV}$ ) was upgraded to a dual Ring Imaging system (RICH), which 
provides full separation between charged pions, kaons and protons over essentially the entire momentum range of the experiment. With the installation of a proton recoil detector in 2006, the identification of hard exclusive reactions has been greatly improved.

\section{Inclusive spin asymmetries}

The world data on polarised inclusive deepinelastic scattering are shown in Fig. 1 at their measured $Q^{2}$ for proton and deuteron targets. It becomes immediately clear that quarks contribute in the medium and valence $x$-region to the spin of the nucleon while there is no indication for significant contributions from sea quarks at small $x$. Although the structure function $g_{1}$

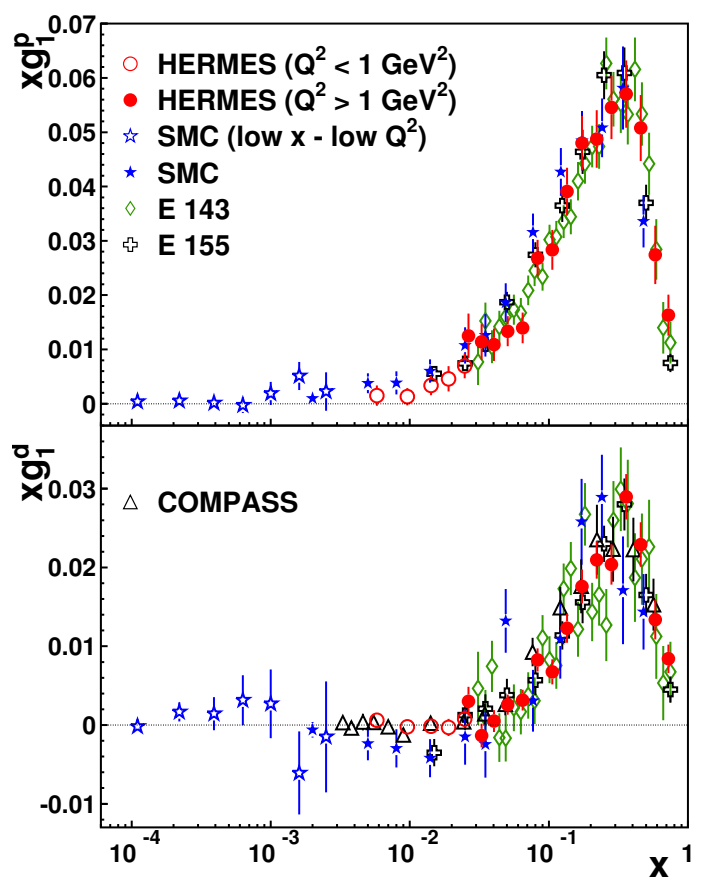

Figure 1. The world data on the spin structure function $x g_{1}(x)$ for the proton and the deuteron show consistent results. The precise deuteron data yield the so far most precise value for the spin contribution of quarks in the nucleon. represents the sum over all quark flavours, the difference between $g_{1}$ for the proton and for the deuteron indicates that up and down quarks have opposite polarisations.

The HERMES data provide the so far most precise determination of $g_{1}(x)$ of the deuteron. Because of isospin symmetry, the data on the deuteron alone alow to extract $\Delta \Sigma$ in the $\overline{M S}$ scheme using only the octed charge $a_{8}$ as additional input

$\Delta \Sigma=\frac{1}{\Delta C_{S}}\left[\frac{9 \Gamma_{1}^{d}}{1-\frac{3}{2} \omega_{D}}-\frac{1}{4} a_{8} \Delta C_{N S}\right]$.

Here $\Delta C_{s}$ and $\Delta C_{N S}$ are QCD coefficients, $\omega_{D}$ is the $\mathrm{D}$-wave contribution in the deuteron wave function and the axial charge $a_{8}$ is obtained from the hyperon beta decay constants assuming $\mathrm{SU}(3)$ flavour symmetry. $\Gamma_{1}^{d}$ is the integrated spin structure function of the deuteron. Fig. 2 shows the cumulative integral of $g_{1}^{p, d, n, N S}$ as a function of the lower integration limit in $x$. For

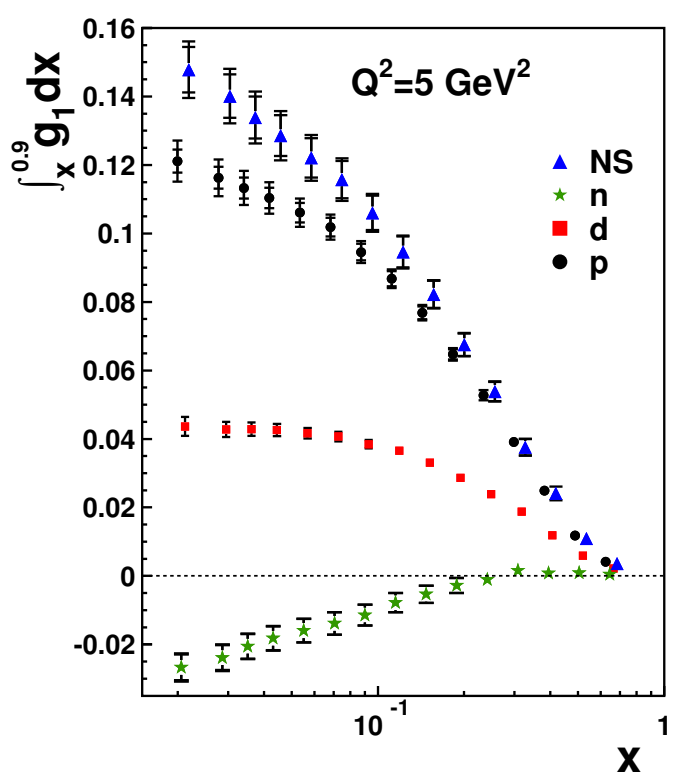

Figure 2. HERMES data: the integrated value of the $g_{1}$ function approaches a constant value at small $\mathrm{x}$ for the deuteron moment (red points). 
$x<0.04, g_{1}^{d}(x)$ becomes compatible with zero (which is also confirmed by the COMPASS data in Fig. 1) and hence its measured integral saturates. Also, the partial first moment of $g_{1}^{d}(x)$ calculated over the range $0<x<0.021$ from a NLO QCD fit of all available data, used here for evolution of the data to a fixed $Q_{0}^{2}$, was found to be consistent with zero. Under this assumption of saturation, the contribution of quark spins to the spin of the nucleon in the $\overline{M S}$ scheme at $Q_{0}^{2}=5 \mathrm{GeV}^{2}$ is $\Delta \Sigma=0.330 \pm 0.011$ (theo) \pm $0.025(\exp ) \pm 0.028$ (evol). This value compares well with the most recent COMPASS result $\Delta \Sigma=$ $0.35 \pm 0.03$ (stat) \pm 0.05 (syst) obtained in the same scheme at $Q_{0}^{2}=3 \mathrm{GeV}^{2}[2]$. The data therefore suggest that the quark helicities contribute a substantial fraction to the nucleon helicity, but there is still need for a major contribution from gluons and/or orbital angular momenta of more than half of the sum of Eq. 1.

\section{Quark helicity distributions}

Semi-inclusive deep-inelastic scattering is a powerful tool to determine the separate contributions $\Delta q_{f}(x)$ of the quarks and antiquarks of flavour $f$ to the total spin of the nucleon. By means of the technique of flavour tagging, individual spin contributions can be determined directly from spin asymmetries of hadrons with the appropriate flavour content. A combined analysis of inclusive and semi-inclusive double-spin asymmetries in the cross section for charged pions and kaons has been carried out for longitudinally polarised hydrogen and deuterium targets [3]. Fig. 3 presents the results from the 5-flavour fit wich are compared with two LO QCD fits $[4,5]$ to all available inclusive data. These fits assume not only $\mathrm{SU}(3)$ flavour symmetry to incorporate hyperon beta decay data, but also explicit symmetry among the three sea quark distributions. The helicity distributions $\Delta u(x)$ and $\Delta d(x)$ extracted from the HERMES data are consistent with the LO QCD fits. The sea quark helicity distributions, extracted separately here for the first time, are consistent with zero and with each other. In particular, no indication was found for a negatively polarised strange sea as expected from anal-

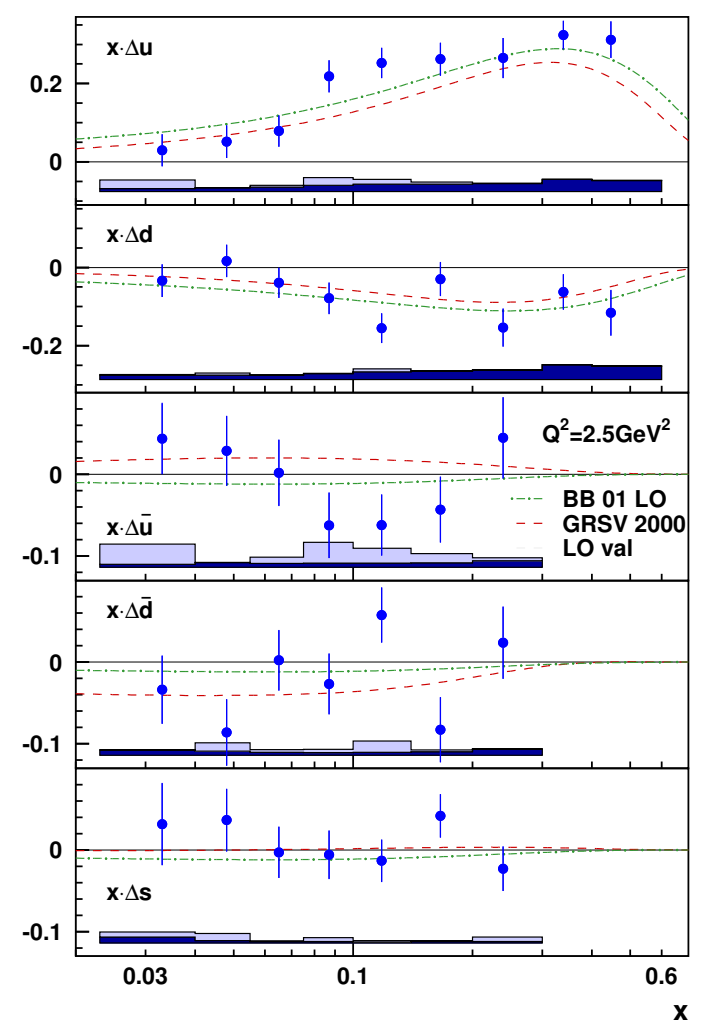

Figure 3. HERMES results for the quark helicity distributions $x \Delta q(x)$ evaluated at a common value of $Q_{0}^{2}=2.5 \mathrm{GeV}^{2}$. The curves show $\mathrm{LO}$ QCD analyses of polarised inclusive data.

yses of only inclusive data assuming SU(3) symmetry applied to hyperon beta decay data.

In a recent analysis [6] of charged kaon production on an isoscalar target, HERMES has extracted the strange quark momentum $S(x)=$ $s(x)+\bar{s}(x)$ and helicity $\Delta S(x)=\Delta s(x)+\Delta \bar{s}(x)$ distributions. This analysis is based only on the assumption of charge conjugation invariance in the fragmentation process and isospin symmetry between proton and neutron. Since strange quarks carry no isospin, the strange PDFs can be assumed to be identical for both nucleons. The fragmentation process in turn can be described by fragmentation functions which have 
no isospin dependence. Fig. 4 shows the strange parton distribution $x S(x)$ extracted from the measured charged kaon multiplicities and taking the value $\int D_{S}^{K}(z) d z=1.27 \pm 0.13$ from the most recent global analysis of fragmentation functions [7]. The results are presented together with

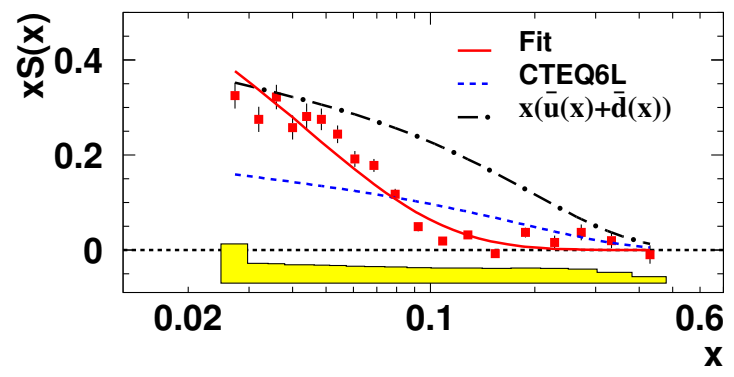

Figure 4 . The strange parton distribution function extracted from the measured HERMES multiplicities for charged kaons and evolved to $Q_{0}^{2}$ $=2.5 \mathrm{GeV}^{2}$. The solid curve is a 3 -parameter fit for $S(x)=x^{-0.924} e^{-x / 0.04}(1-x)$, the dashed curve gives $x S(x)$ from CTEQ6L, and the dotdashed curve is the sum of light antiquarks from CTEQ6L. The substantial deviation of the observed shape of $x S(x)$ from that of the light sea quarks is a clear manifestation of violation of $\mathrm{SU}(3)$ symmetry in the strange quark sector.

(as an example) parametrisations of $x S(x)$ and $x(\bar{u}(x)+\bar{d}(x))$ from CTEQ6L. The shape of $x S(x)$ implied by the HERMES data is incompatible with that from CTEQ6L and other global QCD fits of PDFs as well as with the assumption of an average of an isoscalar nonstrange sea which is a clear manifestation of violation of $\mathrm{SU}(3)$ symmetry in the strange quark sector.

In order to extract the strange quark helicity distribution the measured inclusive and semiinclusive (charged kaon) double-spin asymmetries are used along with the above value for the strange fragmentation function. Fig. 5 shows the simultaneously extracted non-strange $\Delta Q=$ $\Delta u(x)+\Delta \bar{u}(x)+\Delta d(x)+\Delta \bar{d}(x)$ and strange quark $\Delta S=\Delta s(x)+\Delta \bar{s}(x)$ helicity distributions. The results are compared to parametrisations of

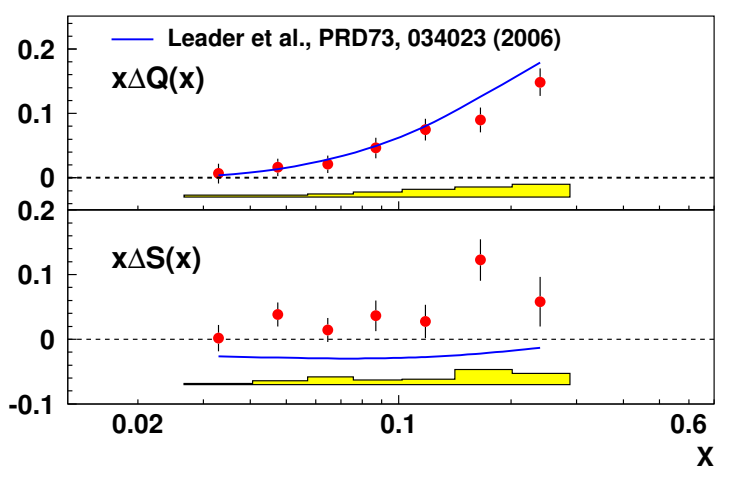

Figure 5. The non-strange (upper panel) and strange quark (lower panel) helicity distributions at $Q_{0}^{2}=2.5 \mathrm{GeV}^{2}$. The curves are a $\mathrm{LO}$ result from a QCD fit of inclusive data. A negative strange quark polarisation is clearly disfavoured by the data.

$\Delta Q$ and $\Delta S$ from a global QCD fit of PDFs to polarised inclusive DIS data assuming $\mathrm{SU}(3)$ symmetry. Clearly, a negative strange quark polarisation is disfavoured by the data. The value for the partial (over the $x$-range 0.02-0.6) octed combination $\Delta q_{8}(x)=\Delta Q(x)-2 \Delta S(x)$ of $0.285 \pm 0.073$ (tot) is substantially less than the value of the axial charge $a_{8}=\Delta q_{8}=0.586 \pm 0.031$ extracted from the hyperon decay constants by assuming $\mathrm{SU}(3)$ symmetry. Possible reasons for the low value of $\Delta q_{8}$ could be either a missing contribution from low $x$ (below 0.02 ) or a violation of $\mathrm{SU}(3)$ symmetry.

Recently, also COMPASS [8] presented an extraction of the strange helicity distribution from charged kaon production on a deuterium taget. The results are consistent with those from HERMES, extending the kinematic range in $x$ down to 0.004 . Together with the data collected with a longitudinally polarised proton target in 2007 , 
COMPASS will be able to also perform a full five flavour separation.

A fully complementary direct measurement of the up- and down quark and anti-quark helicity distributions will be performed at RHIC making use of the maximal parity violation of the $W$ bosons [9]. Such measurements will be free from any systematic uncertainty due to the fragmentation process. They require the running of RHIC at a centre-of-mass energy of $\sqrt{s}=500 \mathrm{GeV}$ with at least $300 \mathrm{pb}^{-} 1$ of luminosity as projected for 2010-2013.

\section{The role of gluons}

In view of the small contribution of quarks to the nucleon spin, an understanding of the role of gluons and the determination of their polarisation has become a major focus of the field. A large polarisation of gluons was expected from investigations of the QCD axial anomaly that tried to attribute the lacking spin contribution by quarks to $Q^{2}$ dependent contributions by gluons.

In principle, a clean determination of the gluon polarisation $\Delta g(x)$ is possible by investigating scaling violations of the spin-dependent structure function $g_{1}\left(x, Q^{2}\right)$. However, the lack of a polarised lepton-proton collider limits the kinematic range of $g_{1}$ to the fixed target domain at moderate $x$ and $Q^{2}$ and $\Delta g$ has been left essentially unconstraind. The way to further proceed is more direct measurements of the gluon polarisation.

In lepton-nucleon scattering, the photon-gluon fusion process gives rise to a double-spin cross section asymmetry proportional to the gluon polarisation

$A_{\|}=R_{p g f} a_{L L}^{p g f} \frac{\Delta g}{g}+A_{b g}$

where $R_{p g f}$ is the fraction of photon-gluon fusion events with an analysing power $a_{L L}^{p g f}$ and $A_{b g}$ is the contribution of processes to $A_{\|}$which are not sensitive to the gluon polarisation. Final states that select the photon-gluon fusion process are heavy-flavour production, $l p \rightarrow c \bar{c} X$, and single- or di-hadron production, $l p \rightarrow h X$ or $l p \rightarrow h_{1} h_{2} X$, where the hadrons have large transverse momentum. Heavy-flavour production is the golden, almost background free $\left(R_{p g f} \approx 1\right)$, channel to access the gluon polarisation, but the low production cross section and the detection of, e.g. open charm $(D-$ mesons $)$, make these investigations experimentally very challenging.

Experimentally much easier is the selection of high $-p_{T}$ hadrons, but the extraction of $\Delta G$ from the measured asymmetry following Eqn. 3 becomes model dependent. Fig. 6 shows the longitudinal double-spin asymmetry for positive and negative hadrons produced inclusively from hydrogen and deuterium targets measured by HERMES. The sensitivity to the gluon polarisation is

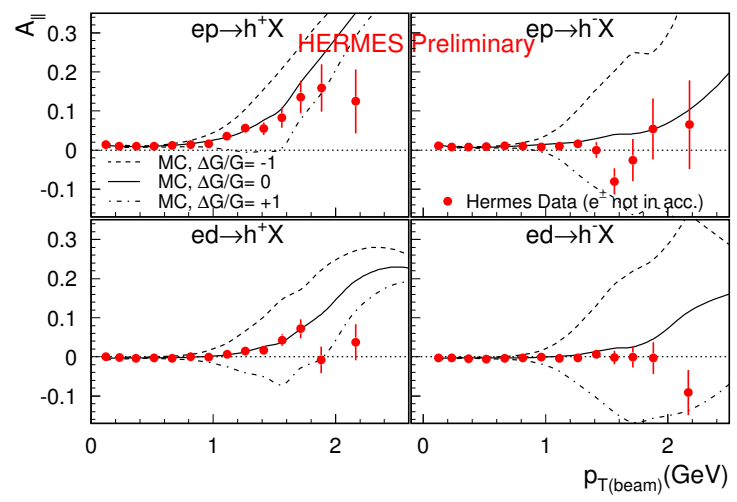

Figure 6 . The longitudinal double-spin asymmetry for positive and negative hadrons produced inclusively from hydrogen and deuterium targets is sensitive to the polarisation of gluons at large values of $p_{T}$. The curves are MC asymmetries calculated for three different assumptions on the gluon polarisation.

enhanced at large values of the transverse momentum of the hadron. Also shown in the figure are the asymmetries expected from the model using the assumptions $\Delta g / g=-1,0$ and +1 (lines from top to bottom) over the covered $x-$ range. The gluon polarisation was extracted by using information on the background aymmetries and the subprocess kinematics obtained from the 
PYTHIA 6.2 leading order Monte Carlo model. The signal processes, photon-gluon fusion and gluon initiated $2 \rightarrow 2$ (resolved-photon) processes, typically contribute only about $20 \%$ to the selected high $-p_{T}$ range. The signal asymmetry contains a convolution of $\Delta g(x) / g(x)$ with the hard subprocess cross section over the $x$-range covered by the data. Two methods have been applied to extract the average $\langle\Delta g / g\rangle\left(p_{T}\right)$ from this asymmetry using different assumptions on the shape of $\Delta g(x) / g(x)$. Method I assumes that $\Delta g(x) / g(x)$ is constant in the relevant $x$-range, while in Method II a functional form is assumed for $\Delta g(x) / g(x)$ which is used to calculate the integral of the signal asymmetry in each $p_{T}$ bin. The results are shown in Fig. 7 for both Methods. For

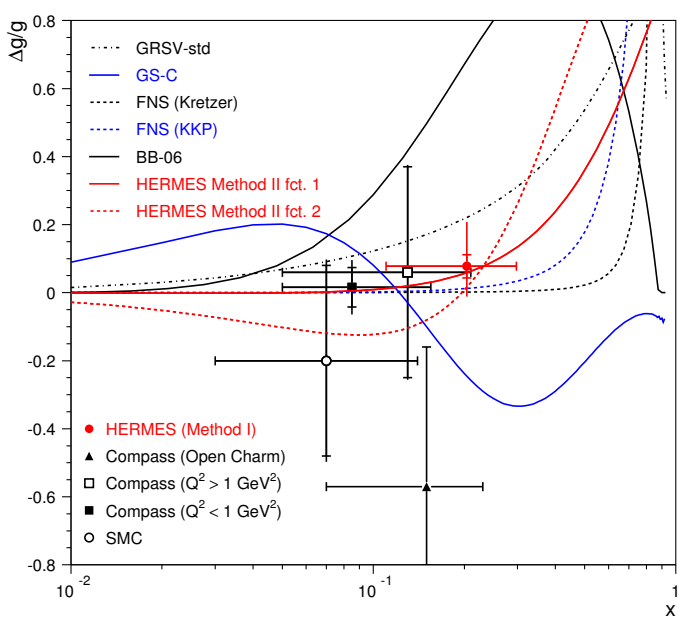

Figure 7. The world data on $\Delta g / g$ extracted by DIS experiments favour surprisingly a very small value for the gluon polarisation in the very restricted $x$-range covered so far. The curves illustrate our lack of knowledge about the polarisation of gluons (see text).

Method II the functional form was varied, given by the two red lines. The experimental systematic error is approximately $15 \%$ and arises from the uncertainties in the beam and target polarisation measurements. It is small compared to the model uncertainty which was estimated by varying the PYTHIA model parameters, the polarised and unpolarised PDFs in the Monte Carlo generation and asymmetry calculation, respectively, and the asymmptions used for the asymmetries of the background processes. No uncertainty was assigned neither on the PYTHIA model itself, nor, because this is a leading order approach, for NLO corrections.

Fig. 7 also summarises all results from photongluon fusion in leptoproduction including the results from the SMC [10] and COMPASS [11] experiments. The curves, showing several different parametrisations of $\Delta g(x) / g(x)$ obtained from NLO QCD fits $[4,5,12,13]$ to $g_{1}$, illustrate the lack of knowledge about the gluon polarisation. In the measured $x$-range, the data favour a very small value for the gluon polarisation, or $\Delta g(x)$ has a node at $x$ around 0.1 .

A crucial issue will be the determination of the gluon polarisation over a wide $x$-range and in particular at small $x$-values. Such measurements are a major focus and strength of RHIC [9]. However, measurements at values of $x<10^{3}$ will only be possible at a high-energy polarised electronproton collider [14].

\section{Transverse spin phenomena and spin- orbit correlations}

Transversity, $h_{1}(x)$ or $\delta q(x)$, describes the distribution of transversely polarised quarks in a transversely polarised nucleon. It is a leading twist structure function of equal importance as $F_{2}(x)$ or $g_{1}(x)$. In the non-relativistic limit $h_{1}(x)$ and $g_{1}(x)$ are identical as both functions describe the orientation of the quark spins relative to the nucleon spin, $g_{1}(x)$ in the helicity basis and $h_{1}(x)$ in a basis of transverse spin eigenstates. However, transversity and helicity densities differ because quarks inside the nucleon move relativistically, hence boosts and rotations do not commute. They are therefore independent quantities which probe different QCD properties. Any experimental evidence for a deviation of $h_{1}(x)$ from $g_{1}(x)$ would be a measure of relativistic effects. Fur- 
thermore, the QCD evolution of $h_{1}\left(x, Q^{2}\right)$ is of special importance as - in contrast to the evolution of $g_{1}$ or $F_{2}$ - gluons do not contribute.

Viewed in the helicity basis, transversity is related to a forward scattering amplitude involving a helicity flip of both quark and target nucleon. Due to this chiral-odd nature, transversity - unlike the other two basic distribution functions cannot be measured in inclusive DIS but only in a process in which it combines with another chiral-odd quantity. The most direct approach is to measure double transverse-spin asymmetries in polarised Drell-Yan processes which couples two transversity distributions. This approach is however experimentally not yet feasible. Another possibility is the semi-inclusive DIS process where fragmentation functions enter the cross section in conjunction with the distribution functions. Such a mechanism has been proposed by Collins [15] where the chiral-odd Collins fragmentation function relates the transverse polarisation of the struck quark with the transverse momentum $P_{h \perp}$ of the produced hadron. This mechanism manifests itself in a single-spin asymmetry, i.e. a left-right asymmetry in the production of hadrons in the plane transverse to the direction of the virtual photon.

Single-spin asymmetries in semi-inclusive DIS on a transversely polarised target can also originate from the Sivers mechanism [16]: it emerges from the combination of the ordinary spinaveraged fragmentation function with the Siver distribution function. This naive time-reversal odd function, i.e. time-reversal without interchange of initial and final state, can be related to the interference of wave functions for different orbital momentum states. As such it parametrises the correlations between the transverse momentum of quarks and the spin of the transversely polarised nucleon. The information on these spinorbit correlations will be a key to construct a complete picture of the internal structure of hadrons beyond the collinear approximation. Both mechanism were studied at HERMES using a transversely polarised hydrogen target. The presented results include the full data set recorded with transverse target polarisation in the years 20022005 [17]. The Collins amplitude $\left\langle\sin \left(\phi+\phi_{S}\right)\right\rangle_{U T}^{h}$ and the Sivers amplitude $\left\langle\sin \left(\phi-\phi_{S}\right)\right\rangle_{U T}^{h}$ have distinct angular signatures and were estracted simultaneously using maximum likelihood fits. The fits also allowed for other possible sine modulations coming from the spin-dependent cross section.

Fig. 8 shows the Collins amplitudes for pions. These results are milestones in the field as the

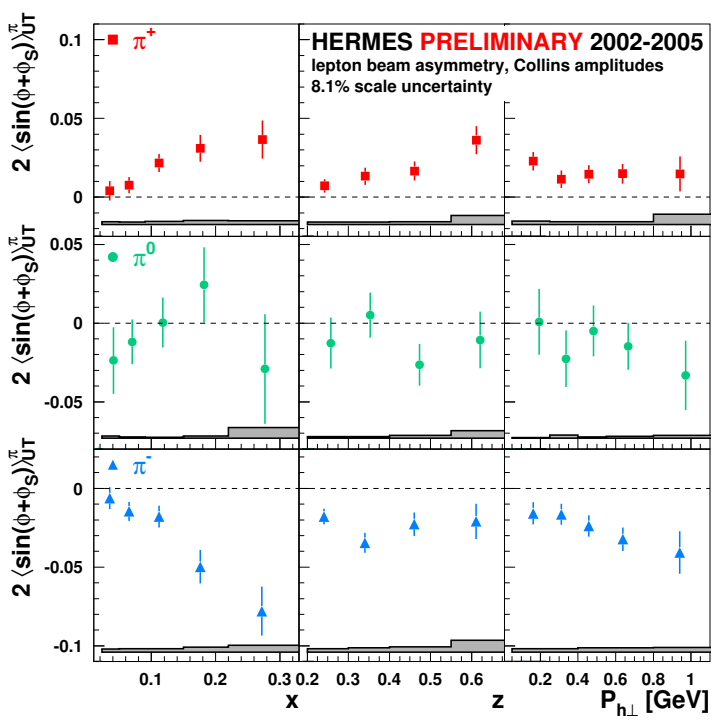

Figure 8. Most recent HERMES results for the Collins amplitudes for $\pi$-mesons. The strikingly non-zero amplitudes for charged pions demonstrated for the first time that both transversity and the Collins fragmentation function exist and are sizeable.

significant non-zero asymmetries for charged pions demonstrated for the first time unambiguously that both the transversity distribution and the Collins fragmentation function exist and are non-zero! The average Collins amplitude is positive for $\pi^{+}$and negative for $\pi^{-}$with a magnitude for $\pi^{-}$comparable or even larger than that of the $\pi^{+}$results, which leads to the conclusion that the disfavoured Collins fragmentation function has a substantial magnitude with opposite 
sign to the favoured one. In combination with further information from the unpolarised process $e^{+} e^{-} \rightarrow h_{1} h_{2} X$ studied at BELLE [18] the transversity distribution function and the Collins fragmentation function for $u$ and $d$ quarks has been extracted for the first time [19].

The $\pi^{0}$ Collins moments represent an important check of isospin symmetry in the $\pi$-meson triplet, which is confirmed by the results. For charged kaons (not shown) no significantly nonzero Collins amplitudes were found.

Fig. 9 shows the Sivers amplitudes for pions and Fig. 10 for charged kaons compared with the pion results. Also these results constitute

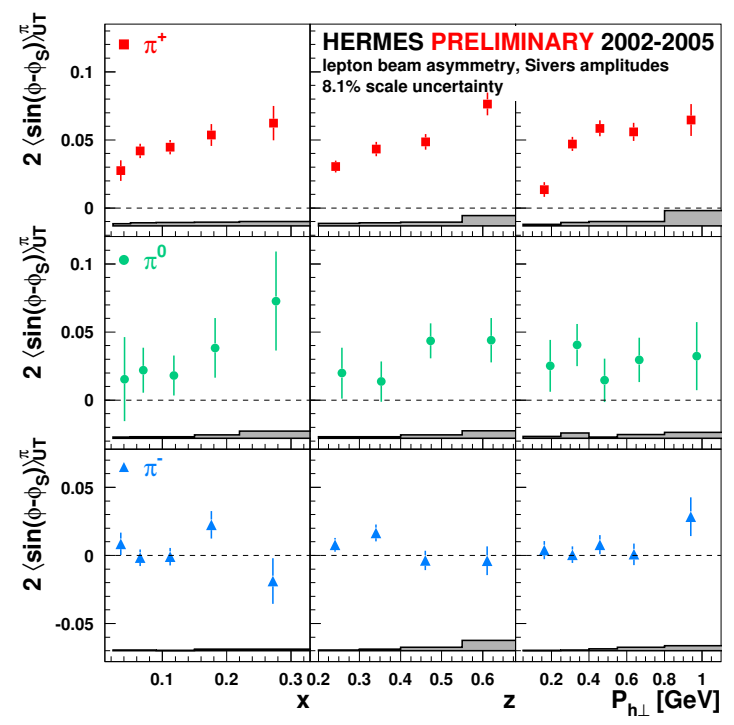

Figure 9. HERMES results for the Sivers amplitudes for $\pi$-mesons. The significant non-zero asymmetries for $\pi^{+}$demonstrated for the first time that T-odd distribution functions indeed exist in DIS. The Sivers function describes spinorbit correlations of the partons in the nucleon.

milestones in the field as the significant non-zero asymmetries for $\pi^{+}, \pi^{0}$ and $K^{+}$demonstrated for the first time that $\mathrm{T}$-odd distribution functions indeed exist in DIS.

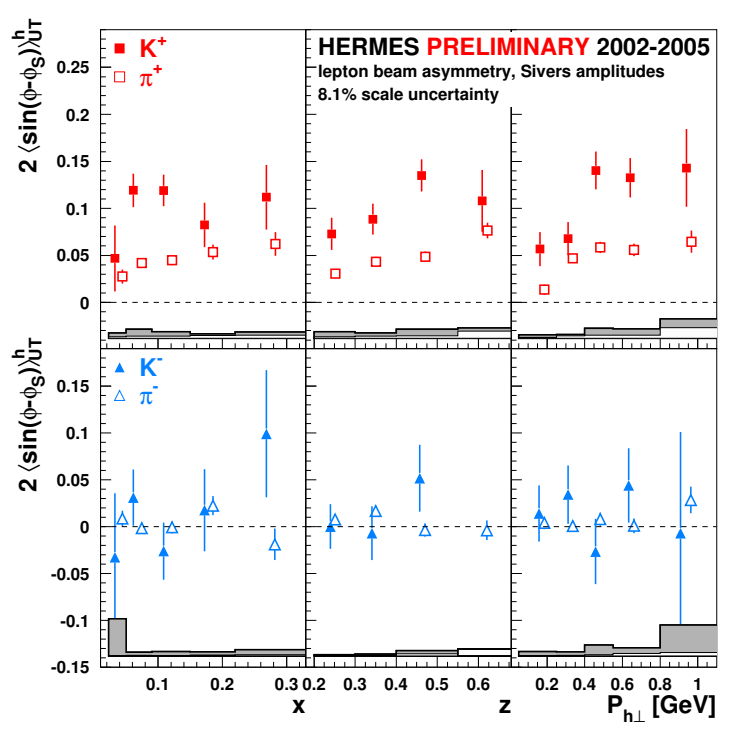

Figure 10. HERMES results for the Sivers amplitudes for charged kaons compared with the charged pion results. The large $K^{+}$moments suggest a significant Sivers function for antiquarks in the nucleon.

Moreover, the existence of such functions depending on transverse momentum of the quarks inside the nucleon implies that these quarks also carry non-vanishing orbital angular momentum which is one of the still missing pieces in the spin puzzle. A direct relation, however, between the Sivers function or other similar functions that describe spin-orbit correlations and the angular momentum contribution of the quarks to the nucleon spin could not yet be established. First, promising attempts of finding such, still model dependent, relations have been made in [20] and [21].

Very interesting are the observed large Sivers amplitudes for $K^{+}$which are roughly twice as large as that for $\pi^{+}$. Since the quark content of these two mesons differ only on the antiquark involved, this observation suggests a significant Sivers function for antiquarks in the proton. Going even a step further, it might also suggest that antiquarks carry significant orbital angular momentum in the nucleon. 
The most crucial test of our current understanding of azimuthal single-spin asymmetries in terms of pertubative QCD will be the experimental verification of the predicted sign change of $\mathrm{T}-$ odd distribution functions, like the Sivers function, when being measured in DIS or in Drell-Yan processes. The experimental check of this prediction is planned by COMPASS when running in the hadron-beam mode and by the proposed PAX experiment at the future FAIR facility at GSI [22].

\section{Prospects of spin physics}

Exciting new information has been obtained on the nucleon spin structure, but a detailed decomposition of the spin of the nucleon remains elusive. One of the most important issues in spin physics remains a detailed measurement of the gluon polarisation.

Transverse spin physics turns out to be a very active and quickly developing field. Important results comprise the first extraction of the transversity as well as of transverse momentum dependent distribution and fragmentation functions like the Sivers distribution and the Collins fragmentation function. These achievements can be considered as milestones in the field. They constitute the first step towards a complete description of the partonic structure of hadrons beyond the collinear parton model.

A rich future is expected for the elegant concept of generalized parton distributions (GPDs). Intensive experimental efforts have demonstrated the feasibility of measurements of hard-exclusive reactions in a large variety of channels [1]. The interplay between spin degrees of freedom and parton orbital angular momentum will be a key to understand the spin structure of the nucleon.

\section{REFERENCES}

1. M. Düren, Hard exclusive reactions and Generalised Parton Distributions at HERMES, proceedings to this Workshop;

2. E.S. Ageev et al. [COMPASS Collaboration], Phys. Lett. B 647 (2007) 8.

3. A. Airapetian et al. [HERMES Collabora- tion], Phys. Rev. Lett. 92 (2004) 012005; Phys. Rev. D 71 (2005) 012003.

4. J. Blümlein and H. Böttcher, Nucl. Phys. B 636 (2002) 225.

5. Glück et al., Phys. Rev. D 63 (2001) 094005.

6. A. Airapetian et al. [HERMES Collaboration], Phys. Lett. B 666 (2008) 446.

7. D. de Florian et al., Phys. Rev. D 75 (2007) 114010.

8. R. Windmolders [COMPASS Collaboration], Proc. of the 18th Int. Spin Physics Symposium (SPIN 2008), Charlottesville, October 2008.

9. G. Bunce et al., Annu. Rev. Nucl. Part. Sci. 50 (2000) 525.

10. B. Adeva et al.[SMC Collaboration], Phys. Rev. D 70 (2004) 012002.

11. E. Ageev et al. [COMPASS Collaboration], Phys. Lett. B 633, 25 (2006); M. Alekseev et al. [COMPASS Collaboration], submitted to Phys. Rev. Lett., arXiv:0802.3023 [hep-ex].

12. T. Gehrmann and W. Stirling, Z. Phys. C 65 (1995) 461.

13. D. de Florian, G. Navarro and R. Sassot, Phys. Rev. D 71 (2005) 094018.

14. A. Despande et al., Annu. Rev. Nucl. Part. Sci. 55 (2005) 165.

15. J.C. Collins, Nucl. Phys. B 396 (1993) 161.

16. D.W. Sivers, Phys. Rev. D 41 (1990) 83.

17. M. Diefenthaler [HERMES Collaboration], Proc. of the 15th Int. Workshop on DIS and Related Subjects, Munich, April 2007, http://dx.doi.org/10.3360/dis.2007.91.

18. R. Seidl et al. [BELLE Collaboration], Phys. Rev. Lett. 96 (2006) 232002.

19. M. Anselmino et al., Phys. Rev. D 75 (2007) 054032 .

20. M. Burkardt, Phys. Rev. D 66 (2002) 114005.

21. S. Meissner, A. Metz and K. Goeke, Phys. Rev. D 76 (2007) 034002.

22. A. Efremov et al., Phys. Lett. B 612 (2005) 233. 